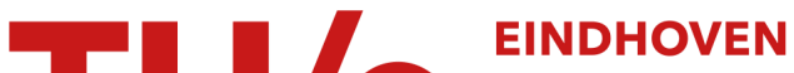 UNIVERSITY OF TECHNOLOGY
}

\section{Experimental Huygens synchronization of oscillators}

\section{Citation for published version (APA):}

Pogromski, A. Y., Rijlaarsdam, D. J., \& Nijmeijer, H. (2010). Experimental Huygens synchronization of oscillators. In M. Thiel, A. Moura, M. C. Romano, \& G. Karolyi (Eds.), Nonlinear dynamics and chaos : advances and perspectives (pp. 195-209). (Understanding complex systems). Springer. https://doi.org/10.1007/978-3-64204629-2_10

DOI:

10.1007/978-3-642-04629-2_10

Document status and date:

Published: 01/01/2010

\section{Document Version:}

Accepted manuscript including changes made at the peer-review stage

\section{Please check the document version of this publication:}

- A submitted manuscript is the version of the article upon submission and before peer-review. There can be important differences between the submitted version and the official published version of record. People interested in the research are advised to contact the author for the final version of the publication, or visit the $\mathrm{DOI}$ to the publisher's website.

- The final author version and the galley proof are versions of the publication after peer review.

- The final published version features the final layout of the paper including the volume, issue and page numbers.

Link to publication

\section{General rights}

Copyright and moral rights for the publications made accessible in the public portal are retained by the authors and/or other copyright owners and it is a condition of accessing publications that users recognise and abide by the legal requirements associated with these rights.

- Users may download and print one copy of any publication from the public portal for the purpose of private study or research.

- You may not further distribute the material or use it for any profit-making activity or commercial gain

- You may freely distribute the URL identifying the publication in the public portal.

If the publication is distributed under the terms of Article 25fa of the Dutch Copyright Act, indicated by the "Taverne" license above, please follow below link for the End User Agreement:

www.tue.nl/taverne

Take down policy

If you believe that this document breaches copyright please contact us at:

openaccess@tue.nl

providing details and we will investigate your claim. 


\title{
Experimental Huygens synchronization of oscillators
}

\author{
Alexander Pogromsky, David Rijlaarsdam, and Henk Nijmeijer \\ Department of Mechanical engineering \\ Eindhoven University of Technology \\ The Netherlands
}

\begin{abstract}
We present an experimental set-up that allows to study both controlled and uncontrolled synchronization between a variety of different type of oscillators. The setup consists of two fully actuated mass-damper-spring oscillators mounted on an actuated platform. By means of different types of feedback realized through computer controlled actuation it is possible to demonstrate different synchronization phenomena, i.e. synchronization of pendula, synchronization of Duffing oscillators, synchronization of rotating bodies, etc. Two experiments are presented where uncontrolled synchronization between two types of identical oscillators is investigated. First, uncontrolled synchronization between two Duffing oscillators is investigated and second, uncontrolled synchronization between two coupled rotating disks is discussed.
\end{abstract}

\section{Introduction}

In the 17th century the Dutch scientist Christiaan Huygens observed a peculiar phenomenon when two pendula clocks, mounted on a common frame, seemed to 'sympathize' as he described it [1]. What he observed was that both clocks adjusted their rhythm towards anti-phase synchronized motion. This effect is now known as frequency or Huygens synchronization and is caused by weak interaction between the clocks due to small displacements of the connecting frame. In [5-8] an extended analysis of this phenomenon is presented. In [9] the authors present an experimental study of Huygens synchronization and finally, in $[10,11]$ a study of the uncontrolled as well as the controlled Huygens experiment is presented.

Many more cases of synchronization have been identified in nature and technology around us [2,3]. Two striking examples in biology are the synchronized flashing of fireflies [4] or synchronization of neurons in the brain when performing perceptual tasks. Other examples of synchronization can be found in $[13,14]$. Using synchronization it is possible to stabilize the frequency of a high power generators and there are more other applications we are unable to mention in this paper.

Three centuries after Huygens the phenomenon of synchronizing driven pendula is, to our best knowledge, repeated twice experimentally. In the

\footnotetext{
* Dedicated to Celso Grebogi at the occasion of his 60th birthday
} 
first research by Bennett, Schatz, Rockwood and Wiesenfeld [5], one has tried to accurately reproduce the findings of Huygens in an experimental setup consisting of two pendulum clocks attached to a free moving cart. The results of this experiment confirm the documented observations of Huygens. A rather simple but interesting experiment is described by Pantaleone [6], where the synchronization of two metronomes is discussed, which are coupled by a wooden board rolling on soda cans. The metronomes in this setup would synchronize most of the time with in phase oscillations.

In this paper we present an experimental set-up [12] that allows to study both controlled and uncontrolled synchronization between a variety of different oscillators. In sections 2 and 3 we outline the goal of the experimental set-up based on our previous experiments. In section 4 the set-up is introduced and we present the dynamical properties of the system. Furthermore, we present the means by which we are able to modify these properties to represent a variety of different oscillators. Next, in section 5 , we present an experiment of the synchronization of two Duffing oscillators. We analyze the stability of the synchronization manifold and continue with numerical and experimental results. Section 6 presents an experiment where the set-up is adjusted to behave like two rotating eccentric discs which are coupled through a third disc mounted on a common axis. Conclusions and future research are presented in section 7 .

\section{Synchronization of pendulum clocks}

Before giving the description of a new experimental set-up we briefly outline experimental studies performed on a simple set-up that consists of two metronomes located on a common beam. Analysis of those experiments motivated us to develop a new set-up that will be described in the subsequent sections. The experimental setup consists of two metronomes coupled by a platform which can translate horizontally. The metronomes are made by Wittner, type Maelzel (series 845). The platform is suspended by leaf springs, which allows a frictionless horizontal translation. A photograph of the experimental setup is given in figure 1 .

Several experiments are performed in order to gain experience with the dynamics of the system. Parameters which can be varied in the experiment are the mass of the platform, the mass and frequency of the metronomes and the amount of damping in the system.

With different parameters the experiments show different phenomena: anti-phase synchronization (see figure 2), an intermediate (neither anti- nor in-phase synchronization) regime with a large amplitude difference of the angles of the metronomes and finally in-phase synchronization (see figure 3), for details, see [9].

Though it was possible to demonstrate different synchronization regimes with such a relatively simple set-up, the experiments revealed difficulties that 
are unavoidable for this set-up. First of all, the experiments showed that the results depend heavily on the escapement mechanism used in the metronomes. This mechanism forces pendulums to oscillate and behave like pendulum clocks. Those mechanisms are relatively difficult to model and their parameters vary for different metronomes causing non-identical synchronization. To change the damping parameter the leaf springs should be replaced and hence the whole set-up should be mechanically rebuilt that resulted in that some experiments are quite difficult to repeat with the same results. Moreover, the set-up allows to perform experiments with only one type of oscillators - pendulums, while Huygens' synchronization can be observed for different types of oscillators, not necessarily pendulums. Analyzing these problems it

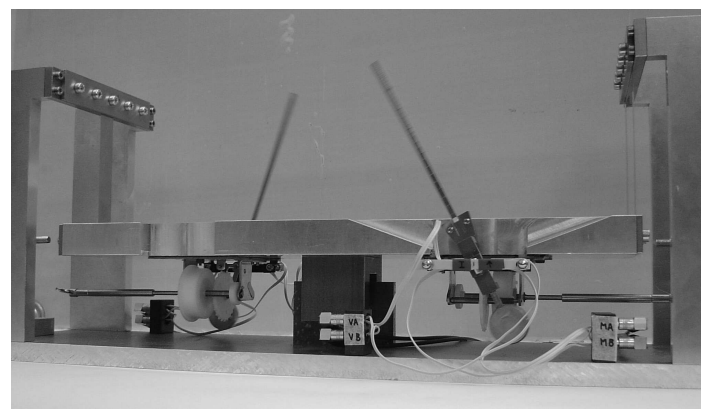

Fig. 1. Photograph of the setup.

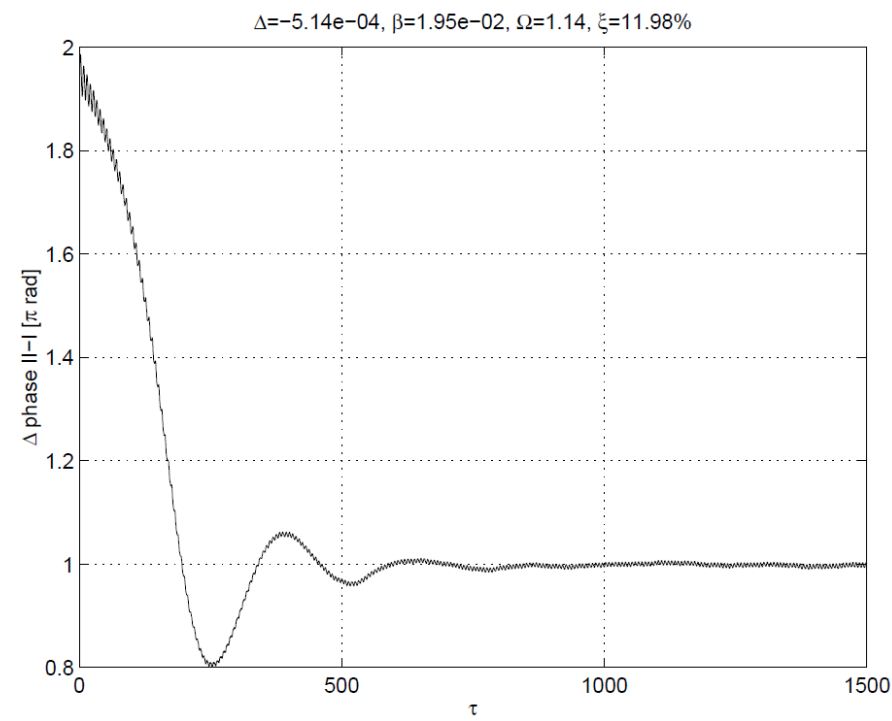

Fig. 2. Anti-phase synchronization. Difference of phases of the oscillators versus time. 
was proposed to design and to build a new synchronization set-up that would be free of those disadvantages.

\section{The goal of the experimental set-up}

Starting from the work of Huygens synchronization phenomena attracted attention of many researchers due to its numerous applications, see e.g. $[13,14,2]$. It turns out that the synchronous behavior can be observed in many practical situations and it demonstrates a certain degree of robustness, that is certain approximate synchronization can happen even if the synchronizing oscillators are not identical. Moreover, understanding of this phenomenon is far from being complete. For example, Christian Huygens observed synchronous behavior of pendulum clocks attached to a common support beam - this effect can be explain by modeling the beam as a one-degree of freedom solid body as is the case in the simple set-up of the previous section, while, to our best knowledge, no attempt was made to analyze this situation taking into account the beam flexibility, reported by Huygens. To discover new aspects of the synchronization phenomenon it would be useful not only to focus on mathematical models and/or computer simulation but also to perform practical experiments to validate the theoretical findings. Moreover, the experiments can also indicate new and interesting directions for theoretical analysis. Since the synchronization can be observed in a variety of different systems it is interesting to have an experimental set-up that can be used to

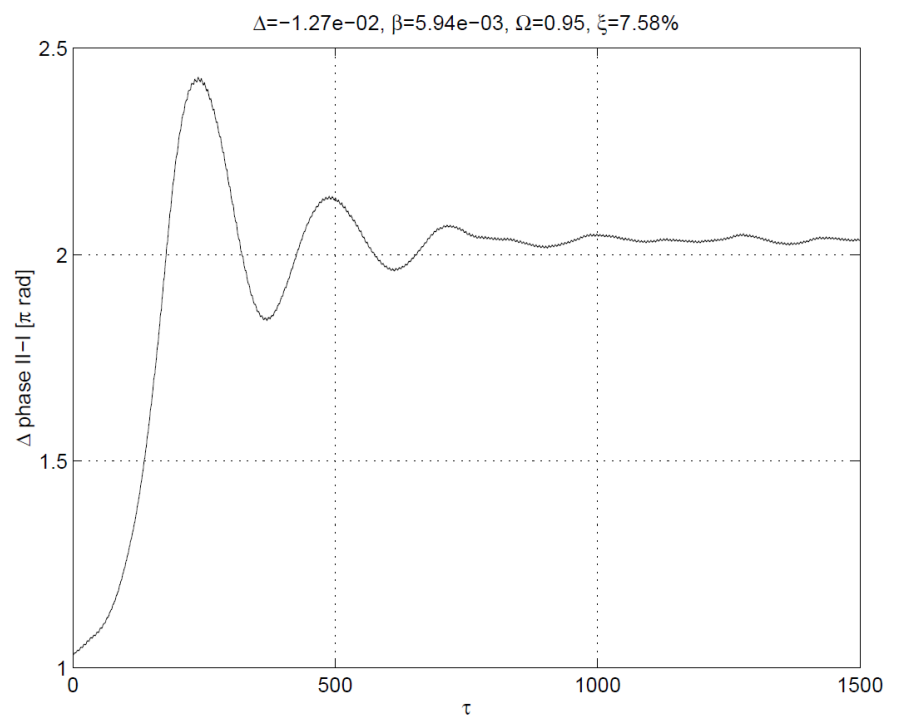

Fig. 3. In-phase synchronization. Difference of phases of the oscillators versus time. 
observe and analyze the synchronous behavior for rather different systems. This set-up is useful for both educational and research purposes.

To meet this objective it was decided to design an electro-mechanical set-up in such a way that its mechanical counterpart is capable to model common features of the synchronizing systems - two one-degree of freedom oscillators mounted on a one-degree of freedom common support body (beam, or platform), while by actuating the oscillators and the beam via computercontrolled feedback it would be possible to mimic a variety of different controlled/uncontrolled synchronizing systems. To this end, the mechanical part was designed as linear as possible, while all the nonlinearities necessary to observe the synchronization are implemented by the electrical part of the set-up. Such a design allows to perform numerous experiments on different systems with limited implementation costs.

The main objectives of the experimental studies are

- To observe experimentally synchronous behavior in a variety of different mechanical systems

- To analyze robustness of the synchronization in a real environment

- To find common features of the synchronizing systems on the uniform experimental platform

\section{The experimental set-up}

In order to experimentally study synchronization between coupled oscillators a set-up consisting of two oscillators, mounted on a common frame has been developed (see figures 4 and 5). The parameters of primary interest

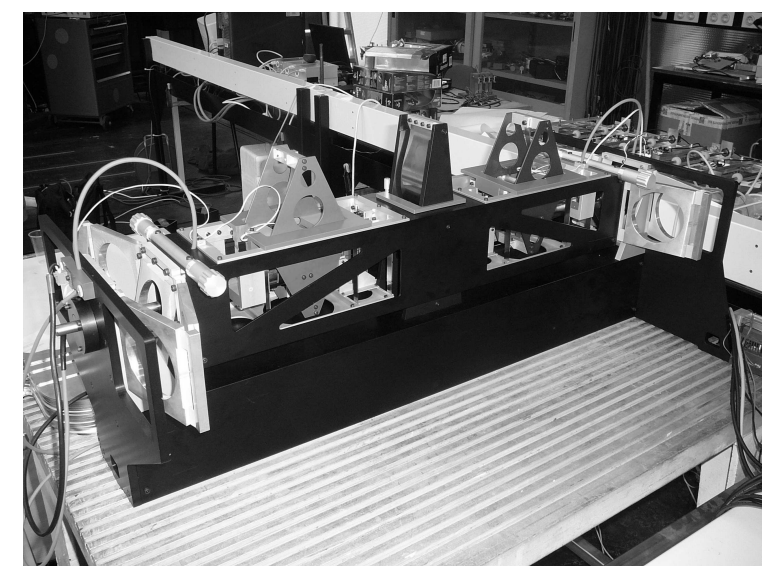

Fig. 4. Photograph of the set-up.

are presented in table 1 . The set-up contains three actuators and position 
sensors covering all degrees of freedom. Furthermore, although the masses of the oscillators $(m)$ are fixed, the mass of the connecting beam $(M)$ may be varied by a factor 10. This allows for mechanical adjustment of the coupling strength. A schematic representation of the set-up is depicted in figure 5 and

Table 1. Parameters in experimental set-up.

\begin{tabular}{|c|c|c|c|}
\hline & Oscillator & $\frac{\text { cillato }}{m}$ & $\frac{\text { ne } / \mathrm{l}}{M}$ \\
\hline $\begin{array}{l}\text { Mass } \\
\text { Stiffness }\end{array}$ & $\begin{array}{c}m \\
\kappa_{1}(\cdot)\end{array}$ & $\begin{array}{c}m \\
\kappa_{2}(\cdot)\end{array}$ & $\begin{array}{c}M \\
\kappa_{3}(\cdot)\end{array}$ \\
\hline Damping & $\beta_{1}(\cdot)$ & $\beta_{2}(\cdot)$ & $\beta_{3}(\cdot)$ \\
\hline
\end{tabular}

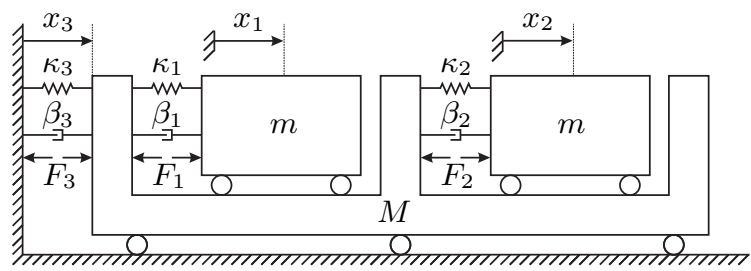

Fig. 5. Schematic representation of the set-up.

the equations of motion of the (ideal) system are:

$$
\begin{aligned}
m \ddot{x}_{1}= & -\kappa_{1}\left(x_{1}-x_{3}\right)-\beta_{1}\left(\dot{x}_{1}-\dot{x}_{3}\right)+F_{1} \\
m \ddot{x}_{2}= & -\kappa_{2}\left(x_{2}-x_{3}\right)-\beta_{2}\left(\dot{x}_{2}-\dot{x}_{3}\right)+F_{2} \\
M \ddot{x}_{3}= & \kappa_{1}\left(x_{1}-x_{3}\right)+\kappa_{2}\left(x_{2}-x_{3}\right) \\
& -\kappa_{3}\left(x_{3}\right)+\beta_{1}\left(\dot{x}_{1}-\dot{x}_{3}\right)+\beta_{2}\left(\dot{x}_{2}-\dot{x}_{3}\right)-\beta_{3}\left(\dot{x}_{3}\right) \\
& +F_{3}-F_{1}-F_{2},
\end{aligned}
$$

where $m, M \in \mathbb{R}_{>0}$ and $x_{i} \in \mathbb{R}, i=1,2,3$ are the masses and displacements of the oscillators and the beam respectively. The functions $\kappa_{i}: \mathbb{R} \mapsto \mathbb{R}, \beta_{i}$ : $\mathbb{R} \mapsto \mathbb{R}$ describe the stiffness and damping characteristics present in the system. $F_{i}$ are the electric actuator forces that may be determined such that the experimental set-up models a large variety of different dynamical systems (see 4.1).

The stiffness and damping in the real system are found to be very well approximated by:

$$
\begin{aligned}
& \kappa_{i}\left(q_{i}\right)=\sum_{j=1}^{5} k_{i j} q^{j} \\
& \beta_{i}\left(\dot{q}_{i}\right)=b_{i} \dot{q}_{i},
\end{aligned}
$$


where $q_{1}=x_{1}-x_{3}, q_{2}=x_{2}-x_{3}$ and $q_{3}=x_{3}$. The values of $k_{i j}$ and $b_{i} \forall i=1,2,3$ have been experimentally obtained and will be used to modify the systems' properties in the sequel.

\subsection{Adjustment of the System Properties}

In order to experiment with different types of oscillators, the derived properties (stiffness and damping) are adjusted. Note that, since we know the damping and stiffness present in the system and since we can fully measure the state of the system, we may adjust these properties, using actuators, to represent any dynamics we want. This allows modeling of different types of springs (linear, cubic) and any other desired effect within the limits of the hardware. In the next part of this paper we present two examples of this type of modulation. The system is first adapted to analyze synchronization between Duffing oscillators and secondly to analyze the synchronizing dynamics of two coupled rotating eccentric discs under the influence of gravity.

\section{Example 1: Coupled Duffing Oscillators}

In this section experimental results with respect to two synchronizing Duffing oscillators are presented. After introducing the dynamical system analysis of the limiting behaviour of the system is presented. Finally, both numerical and experimental results are presented and discussed.

\subsection{Problem Statement and Analysis}

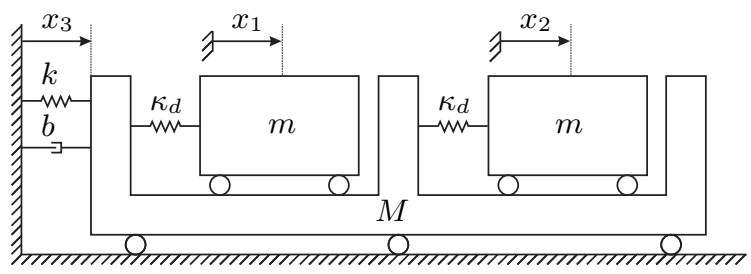

Fig. 6. Schematic representation of the set-up modeling two coupled Duffing oscillators.

Consider the system as depicted in figure 6 , where

$$
\frac{\kappa_{d}\left(q_{i}\right)}{m}=\omega_{0}^{2} q_{i}+\vartheta q_{i}^{3}
$$

where $q_{i}=x_{i}-x_{3}$ and constants $\omega_{0}, \vartheta \in \mathbb{R}_{>0}$. 
The system under consideration represents two undriven, undamped Duffing oscillators coupled through a third common mass. The set-up depicted in figure 5 can be adjusted to model this system by defining the actuator forces as:

$$
\begin{aligned}
& F_{i}=\kappa_{i}\left(q_{i}\right)+\beta_{i}\left(\dot{q}_{i}\right)-\kappa_{d}\left(q_{i}\right), i=1,2 \\
& F_{3}=0
\end{aligned}
$$

Where $F_{3}=0$ is chosen because, in the original set-up, the beam already models the situation as depicted in figure 6 (linear stiffness and damping) fairly accurately. The equations of motion of the resulting system are linear:

$$
\begin{aligned}
& m \ddot{x}_{1}=-\kappa_{d}\left(x_{1}-x_{3}\right) \\
& m \ddot{x}_{2}=-\kappa_{d}\left(x_{2}-x_{3}\right) \\
& M \ddot{x}_{3}=\kappa_{d}\left(x_{1}-x_{3}\right)+\kappa_{d}\left(x_{2}-x_{3}\right)-k x_{3}-b \dot{x}_{3},
\end{aligned}
$$

where $k, b \in \mathbb{R}_{>0}$ are the stiffness and damping coefficients of the beam.

Before continuing with the experimental and numerical results the system's limiting behaviour is analyzed. In order to do so the notion of antiphase synchronization needs to be defined. We call the solutions of $x_{1}(t)$ and $x_{2}(t)$ asymptotically synchronized in anti-phase if they satisfy the following ralation

$$
\lim _{t \rightarrow \infty}\left\|x_{1}(t)-\alpha \sigma_{\left(\frac{T}{2}\right)} x_{2}(t)\right\|=0,
$$

with $\alpha$ a scale factor and $\sigma_{\left(\frac{T}{2}\right)}$ a shift operator over half an oscillation period $(T)$. Respectively, if instead of the previous relation it follows that

$$
\limsup _{t \rightarrow \infty}\left\|x_{1}(t)-\alpha \sigma_{\left(\frac{T}{2}\right)} x_{2}(t)\right\| \leq \varepsilon,
$$

for some small $\varepsilon>0$, we say that the solutions are approximately asymptotically synchronized in anti-phase.

It can been shown that the dynamics of the oscillators in (9) - (11) converges to anti-phase synchronization as $t \rightarrow \infty$.

To prove this claim consider the system (9) - (11). To analyze the limit behaviour of this system, the total energy is proposed as a candidate Lyapunov function:

$$
\mathcal{V}=\frac{1}{2} \sum_{i=1}^{3} m_{i} \dot{x}_{i}^{2}+\sum_{i=1}^{3} \int_{0}^{\xi_{i}} \kappa_{i}(s) d s
$$

where $m_{1}=m_{2}=m, m_{3}=M, \xi_{i}=x_{i}-x_{3}, i=1,2, \xi_{3}=x_{3}, \kappa_{i}\left(q_{i}\right)=\kappa_{d}\left(q_{i}\right)$ and $\kappa_{3}=k x_{3}$. Calculating the time derivative of $\mathcal{V}$ along the solutions of the system (9) - (11) yields:

$$
\dot{\mathcal{V}}=-b \dot{x}_{3}^{2}
$$


Hence, we find $\dot{\mathcal{V}} \leq 0$ and the system may be analyzed using LaSalle's invariance principle.

Equation (15) implies that $\mathcal{V}$ is a bounded function of time. Moreover, $x_{i}(t)$ is a bounded function of time and will converge to a limit set where $\dot{\mathcal{V}}=0$. On this limit set $\dot{x}_{3}=\ddot{x}_{3}=0$, according to (15). Substituting this in system (9) - (11) yields $x_{3}=0$ on the system limit set. Substituting $x_{3}=\dot{x}_{3}=\ddot{x}_{3}=0$ in (11) shows:

$$
\kappa_{d}\left(x_{1}\right)=-\kappa_{d}\left(x_{2}\right)
$$

Since $\kappa_{d}$ is a one-to-one, odd function, this implies:

$$
x_{1}=-x_{2}
$$

Finally, substituting $x_{1}=-x_{2}$ in (9) - (10) yields:

$$
\dot{x}_{2}=-\dot{x}_{1} .
$$

Summarizing, it has been shown that any solution of (9) - (11) will converge to anti-phase synchronized motion according to definition the given definition.

The next paragraph will present numerical and experimental results that support the analysis provided in this section.

\subsection{Experimental and Numerical Results}

In order to experimentally investigate the synchronizing behaviour of two coupled Duffing oscillators the set-up has been modified as specified in the previous section. The oscillators are released from an initial displacement of $-3 \mathrm{~mm}$ and $-2.5 \mathrm{~mm}$ respectively (approximately in phase) and allowed to oscillate freely.

Figure 7 shows the sum of the positions of the oscillators and the position of the beam versus time. As becomes clear from figure 7, approximate antiphase synchronization occurs within $40 \mathrm{~s}$. Furthermore, figure 8 shows the limiting behaviour of both oscillators and the beam. Although the amplitudes of the oscillators differ significantly, the steady state phase difference is $1.01 \pi$. The most probable cause for the amplitude difference is the fact that the oscillators are not exactly identical. As a result, the beam does not come to a complete standstill, although it oscillates with an amplitude that is roughly ten times smaller than that of the oscillators.

In addition to the experimental results, numerical results are provided in figure 9 and 10. The parameters in the simulation are chosen as shown in table 2. The results presented in figure 9 and 10 correspond to the experimental results provided in 7 and 8 respectively. Although the oscillation frequencies of the oscillators are almost equal (within 5\%) in the simulation and the experiment, the final amplitudes of the oscillators differs by a factor 15 . This 

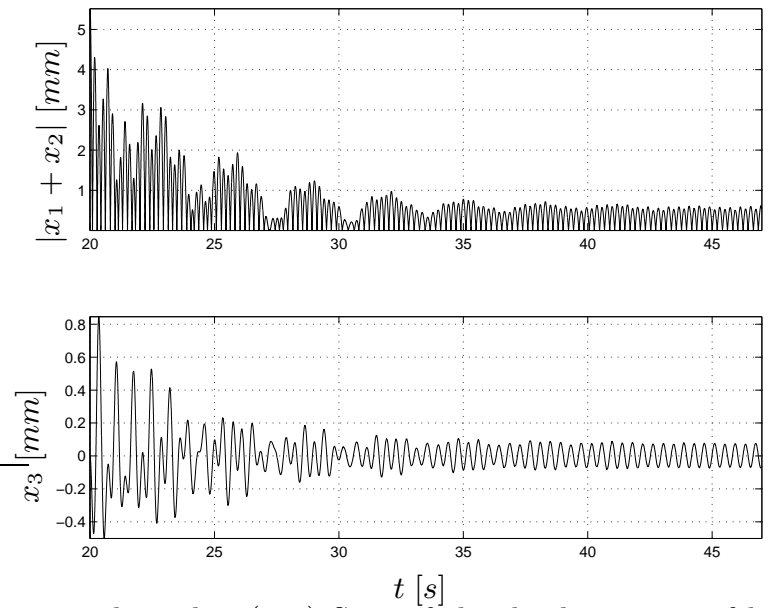

Fig. 7. Experimental results: (top) Sum of the displacements of both oscillators. (bottom) Displacement of the connecting beam.
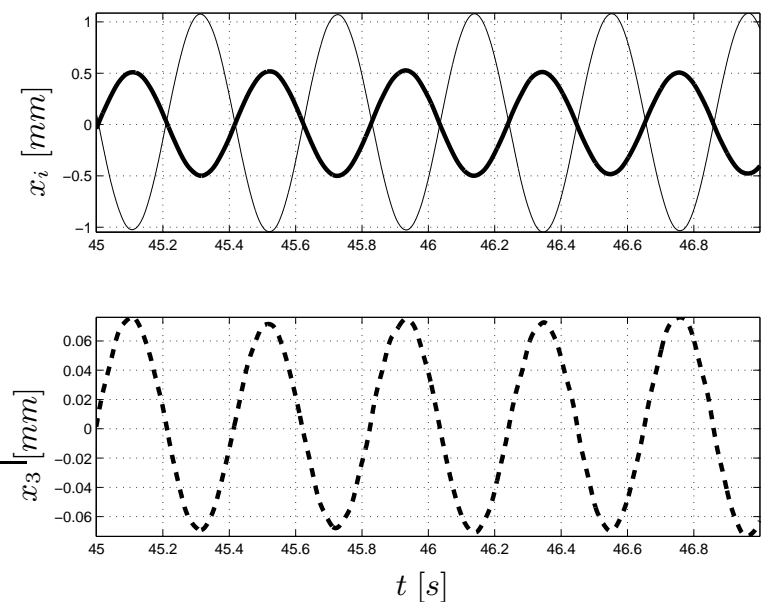

Fig. 8. Experimental results: Steady state behaviour of the system. (top) Displacement of the oscillators $\left(-x_{1},-x_{2}\right)$. (bottom) Displacement of the connecting beam.

is due to the fact that in the experiment the damping is over-compensated, resulting in larger amplitudes of the oscillators. In the numerical simulation almost exact anti-phase synchronization with equal oscillator amplitudes is achieved.

Finally, note that some of the differences between the experimental and simulation results may be coped with by tuning either the parameters of the numerical simulation or those of the set-up itself. The question of identifying 
a model can thus be reversed to tuning the parameters of the set-up rather than those of the model.
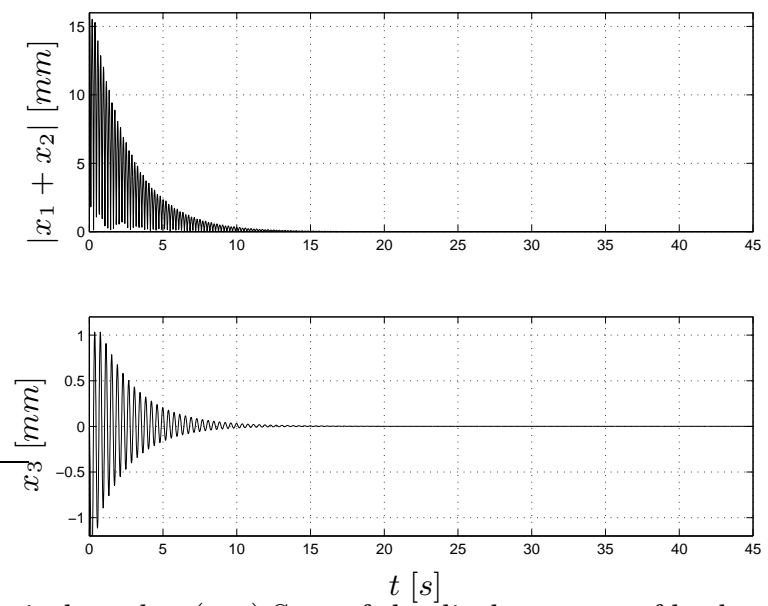

Fig. 9. Numerical results: (top) Sum of the displacements of both oscillators. (bottom) Displacement of the connecting beam.
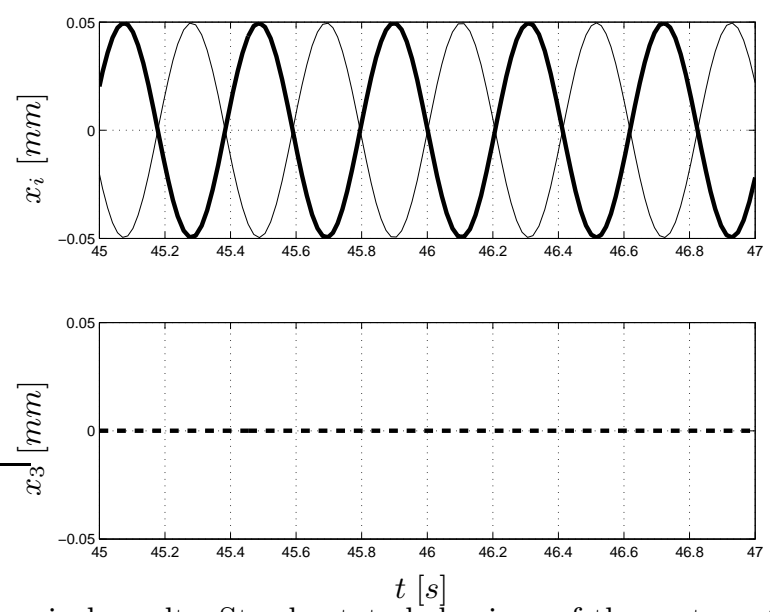

Fig. 10. Numerical results: Steady state behaviour of the system. (top) Displacement of the oscillators $\left(-x_{1},-x_{2}\right)$. (bottom) Displacement of the connecting beam. 


\section{Example 2: Two Coupled Rotary Disks}

Next to the synchronization of Duffing oscillators we investigated synchronization in a system of coupled rotating discs as depicted in figure 11. First the dynamics of the system will be specified in more detail and next experimental results will be presented.

\subsection{Problem Statement}

Consider the system as depicted in figure 11. This system consists of three discs. Discs 1, 2 represent the oscillators and disc 3 is connected to both other discs by torsion springs with stiffness $k$. Each of the discs has an eccentric mass at a distance $\ell_{i}$ from it's center $\left(\ell_{1}=\ell_{2}=\ell\right)$. Furthermore the middle disc is coupled to the world by a torsion spring with stiffness $k_{3}$ and a torsion damper with constant $b$. The rotation of the discs is represented w.r.t. the world by the angles $\theta_{i}$. The equations of motion of the system depicted in figure 11 are:

$$
\begin{aligned}
\ddot{\theta}_{i}= & -\vartheta_{i}\left(k\left(\theta_{i}-\theta_{3}\right)+\delta_{i} \sin \theta_{i}\right), i=1,2 \\
\ddot{\theta}_{3}= & \vartheta_{3}\left(\sum_{j=1}^{2} k\left(\theta_{j}-\theta_{3}\right)\right. \\
& \left.-k_{3} \theta_{3}-b_{3} \dot{\theta}_{3}-\delta_{3} \sin \theta_{3}\right),
\end{aligned}
$$

with $\vartheta_{i}=\frac{1}{m \ell_{i}^{2}+J_{i}}$ and $\delta_{i}=m_{i} g \ell_{i}$. The modification to the set-up is now more involved than in the previous example. First of all, the translation coordinates $x_{i}$ should be mapped to rotation angles $\theta_{i}$. Secondly, in case of the Duffing oscillator the actuation forces $F_{1}$ and $F_{2}$ are meant to act on both the oscillators and the connecting mass. In the situation depicted in figure 11 the actuation force generated to model the coupling between the oscillator discs and the middle disc by means of the torsion spring should again act on the oscillators and the connecting beam in our set-up. However, the part of the actuation force that models the influence of gravity on the oscillators should only act on the oscillators and not on the connecting beam, since in figure 11 the gravity on discs 1 and 2 exerts a force only on the corresponding disc and not directly on the middle mass.

In order to adjust the set-up in figure 5 to model the system in figure 11 the actuator forces are defined as:

$$
\begin{aligned}
& F_{i}=\kappa_{i}\left(q_{i}\right)+\beta_{i}\left(\dot{q}_{i}\right)-\vartheta_{i}\left(\eta_{i}+g_{i}\right), i=1,2 \\
& F_{3}=\kappa_{3}\left(x_{3}\right)-\vartheta_{3}\left(\eta_{3}+g_{3}\right)-\tilde{g}(\cdot),
\end{aligned}
$$

with $\kappa_{i}\left(q_{i}\right)$ and $\beta_{i}\left(\dot{q}_{i}\right)$ as defined $(4,5), \eta_{i}=k\left(\theta_{i}-\theta_{3}\right), i=1,2, g_{i}=\delta_{i} \sin \theta_{i}$ and $\tilde{g}=\sum_{j=1}^{2} \vartheta_{i} g_{i}$. Damping is left to be the natural damping of the beam in 
the set-up. Furthermore, translation is mapped to rotation angles according to: $\theta_{i}=\frac{\pi}{2} \frac{x_{i}}{x_{i}^{\star}}$, with $x_{i}^{\star}$ is the maximal displacement of the oscillators and the beam, assuring $\pm 90^{\circ}$ turns in the rotation space.

\subsection{Experimental Results}

Experimental results, are presented in figures 12 and 13. It becomes clear that approximate anti-phase synchronization occurs after about $20 \mathrm{~s}$, like in the Huygens' pendulum set-up. Again complete synchronization does not occur because the oscillators are not identical. In addition figure 13 shows the steady state behaviour of the rotating system, from which the approximate anti-phase synchronized behaviour becomes immediately clear.

\section{Conclusions}

We presented a set-up capable of conducting synchronization experiments with a variety of different oscillators. Two sets of experimental results were provided that show the potential of this set-up. First we modeled and experimentally obtained synchronization between two coupled Duffing oscillators. Second, we showed that it is possible to model systems with rotating dynamics and to effectively model the local influence of gravity in this case.

In addition to studying uncontrolled synchronization the set-up has the potential to study controlled synchronization. Furthermore, we aim to model the Huygens set-up and perform controlled and uncontrolled synchronization experiments with this type of dynamical system.

\section{Acknowledgement}

This work was partially supported by the Dutch-Russian program on interdisciplinary mathematics "Dynamics and Control of Hybrid Mechanical Systems" (NWO grant 047.017.018).

\section{References}

1. C. Huygens, Oeuvres complétes de Christiaan Huygens, Martinus Nijhoff, vol. $5,17,1893,1932$.

2. S. Strogatz, SYNC. The engineering science of spontaneous order, Hyperion, new York, 2003.

3. A. Pikosvky and M. Rosenblum and J. Kurths, Synchronization, Cambridge University Press, 2001.

4. J. Buck, Synchronous rhythmic flashing of fireflies. II., Quaterly review of biology, vol. 63(3), pp. 265-289, 1988. 
5. M. Bennett and M. Schat and H. Rockwood and K. Wiesenfeld, Huygens's clocks, Proceedings of the Royal Society A: Mathematical, Physical and Engineering Sciences 458(2019), pp. 563-579, 2002.

6. J. Pantaleone, Synchronization of metronomes, American Jounal of Physics, 70(10), pp. 992-1000, 2002.

7. M. Senator, Synchronization of two coupled escapement-driven pendulum clocks, Journal of Sound and Vibration, 291(3-5), pp. 566-603, 2006.

8. N.V. Kuznetsov, G.A. Leonov, H. Nijmeijer and A.Yu. Pogromsky, Synchronization of Two Metronomes, 3rd IFAC Workshop 'Periodic Control Systems', St. Petersburg, Russia, CDROM, 2007.

9. W. Oud, H. Nijmeijer and A.Yu. Pogromsky, A study of Huygens synchronization. Experimental Results, Proceedings of the 1st IFAC Conference on Analysis and Control of Chaotic Systems, France, CDROM, 2006.

10. A.Yu Pogromsky, V.N. Belykh and H. Nijmeijer, Controlled synchronization of pendula, Proceedings 42rd IEEE Conference on Decision and Control, pp. 4381-4368, 2003.

11. A.Yu Pogromsky, V.N. Belykh and H. Nijmeijer, A Study of Controlled Synchronization of Huygens' Pendula, in Group Coordination and Cooperative Control, K.Y. Pettersen J.T. Gravdahl and H. Nijmeijer (eds.), Lecture Notes in Control and Information Sciences, pp. 205-216, Springer, 2006.

12. M.H.L.M. van den Tillaart, Design of a mechanical synchronizing system for research and demonstration purooses for D\&C, Master Thesis, Eindhoven University of Technology, 2006.

13. I.I. Blekhman, Synchronization in science and technology, ASME Press, New York, 1988.

14. W. Lindsey, Synchronization systems in communications and control, PrenticeHall, Englewood Cliffs, NJ, 1972. 
Table 2. Parameters in numerical simulation.

\begin{tabular}{ccc}
\hline$\omega_{o}=15.26$ & $\vartheta=8.14$ & $M=0.8$ \\
$m=1$ & $k=1$ & $b=5$ \\
\hline
\end{tabular}

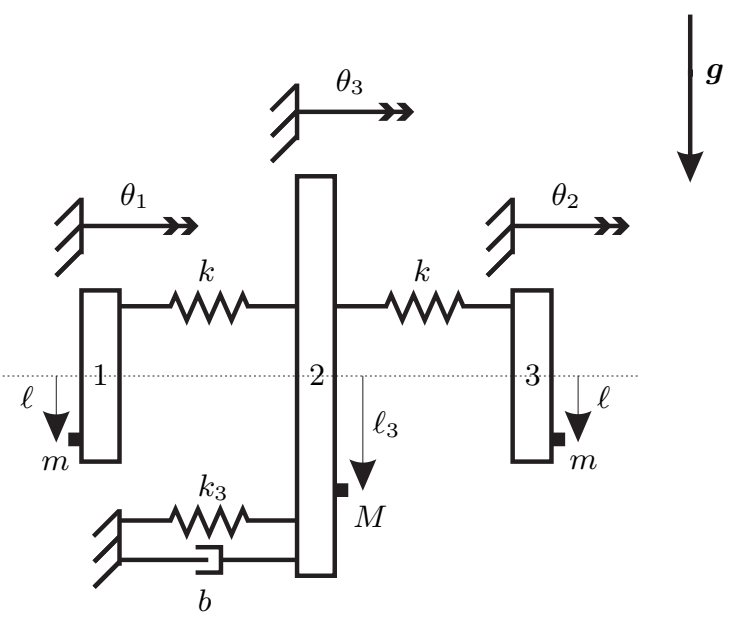

Fig. 11. Schematic representation of the set-up modeling two coupled rotating elements.
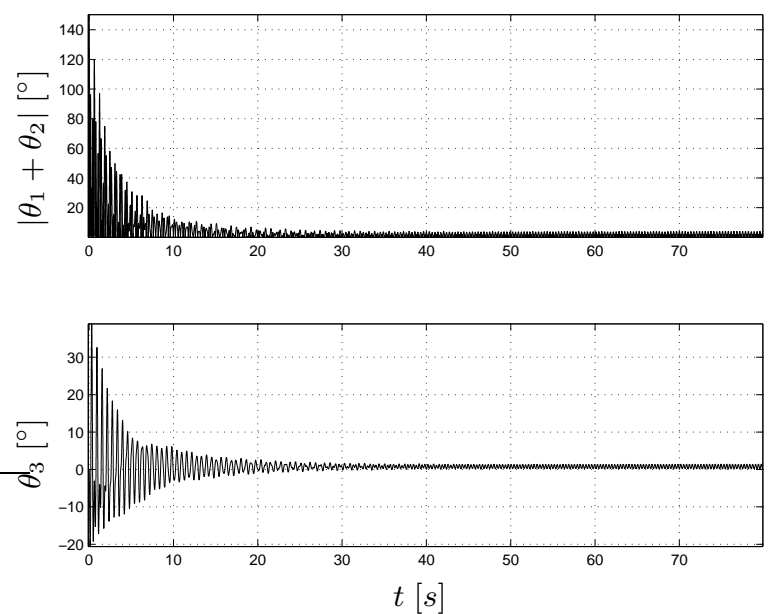

Fig. 12. Experimental results: (top) Sum of the rotation angles of the outer discs. (bottom) Rotation angle of the connecting disc. 

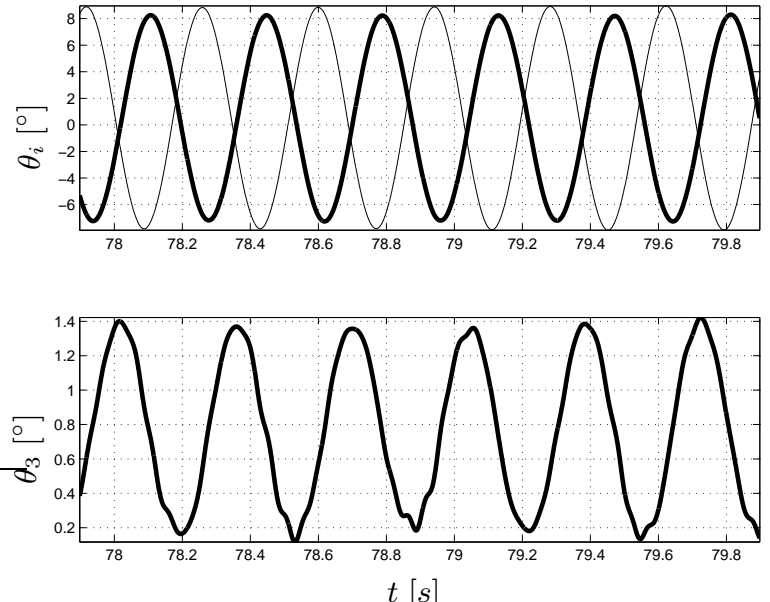

Fig. 13. Experimental results: Steady state behaviour of the system. (top) Outer discs $\left(-\theta_{1},-\theta_{2}\right)$. (bottom) Connecting disc. 